PROCEEDINGS OF THE

AMERICAN MATHEMATICAL SOCIETY

Volume 132, Number 12, Pages 3653-3659

S 0002-9939(04)07513-6

Article electronically published on July 26, 2004

\title{
A LOWER BOUND FOR THE STABILITY RADIUS OF TIME-VARYING SYSTEMS
}

\author{
ADINA LUMINIŢA SASU AND BOGDAN SASU \\ (Communicated by Joseph A. Ball)
}

\begin{abstract}
We introduce and characterize the stability radius of systems whose state evolution is described by linear skew-product semiflows. We obtain a lower bound for the stability radius in terms of the Perron operators associated to the linear skew-product semiflow. We generalize a result due to Hinrichsen and Pritchard.
\end{abstract}

\section{INTRODUCTION}

In the last few years, the theory of linear skew-product semiflows has proved to be a very useful tool in the study of evolution equations with unbounded coefficients (see [4]-[6], 10], 11, 14-20]). Significant questions concerning the asymptotic behaviour of linear skew-product semiflows have been answered. In this context, the theorems of Perron type or so-called "input-output" conditions of characterization of the asymptotic properties, have been obtained for uniform exponential stability (see [19]), for uniform exponential expansiveness (see 16]) and for pointwise and uniform exponential dichotomy, respectively, of discrete and continuous linear skewproduct semiflows (see [4- [6], 10], 11], [15, 17], [18, 20]).

Recently, theorems of Perron type for exponential dichotomy of different types of evolution families have been presented in [12] and in [13. Significant results obtained with input-output techniques have been established in [1]-[3]. An important interpretation which justifies the term "frequency-response function" for time-varying finite-dimensional systems, was given in [3].

It is well known that the concept of stability radius introduced by Hinrichsen, Ilchmann and Pritchard (see 8], [9]) led to a systematic study of the stability of linear infinite-dimensional systems under structured time-varying perturbations. The idea was to estimate the size of the smallest disturbance operator under which the additively perturbed system loses exponential stability. An outstanding result expresses the lower bound of the stability radius of a systems associated to a mild evolution family in terms of the norm of the input-output operator (see 9], Theorem 3.2). This result has been recently proved by Clark, Latushkin, Montgomery-Smith and Randloph (see [7], Theorem 4.2), using an evolution semigroup technique.

Received by the editors April 7, 2003 and, in revised form, August 24, 2003.

2000 Mathematics Subject Classification. Primary 34D05.

Key words and phrases. Linear skew-product semiflows, stability radius.

(C)2004 American Mathematical Society 
Naturally, the question arises whether the concept of stability radius can be extended for the case of systems associated to linear skew-product semiflows, and how can we express the lower bound of this type of stability radius.

The purpose of this paper is to answer this question. We shall consider an abstract generalization of systems described by differential equations of the form

$$
\left\{\begin{array}{l}
\dot{x}(t)=A(\sigma(\theta, t)) x(t)+B(\sigma(\theta, t)) u(t) \\
y(t)=C(\sigma(\theta, t)) x(t)
\end{array}\right.
$$

where $\sigma$ is a semiflow on a locally compact metric space $\Theta$. For every $\theta \in \Theta$, the operators $A(\theta)$ are generally unbounded operators on a Banach space $X$, while the operators $B(\theta) \in \mathcal{B}(U, X), C(\theta) \in \mathcal{B}(X, Y)$, where $U, Y$ are Banach spaces. Formally, in our investigations, the family $\{A(\theta)\}_{\theta \in \Theta}$ is subjected to additive structured perturbations, so that the perturbed system is

$$
\dot{x}(t)=[A(\sigma(\theta, t))+B(\sigma(\theta, t)) \Delta(\sigma(\theta, t)) C(\sigma(\theta, t))] x(t),
$$

which can be interpreted as a system obtained by applying the feedback $u(t)=$ $\Delta(\sigma(\theta, t)) y(t)$ to the time-varying system (1.1).

It is well known that if $X$ is a Banach space, $\Theta$ is a compact metric space, $\sigma$ is a semiflow on $\Theta$ and $A: \Theta \rightarrow \mathcal{B}(X)$ is a continuous mapping, then $\Phi(\theta, t)$, the solution operator of the linear differential equation

$$
\dot{x}(t)=A(\sigma(\theta, t)) x(t), \quad t \geq 0,
$$

is a cocycle and the pair $\pi=(\Phi, \sigma)$ is a linear skew-product semiflow. Therefore, in what follows, we consider an integral model for the above type of systems, defined in terms of linear skew-product semiflows. Our aim is to define and examine a stability radius for a general system associated to a linear skew-product semiflow, subjected to perturbations. The main tools in our study will be the theorems of Perron type, which connect the stability of a linear skew-product semiflow with the uniform boundedness of a family of Perron operators on $C_{b}\left(\mathbf{R}_{+}, X\right)$. We will express the lower bound of the stability radius in terms of the norms of inputoutput operators associated to the system. In this manner, we generalize a result proved by Hinrichsen and Pritchard for the case of mild evolution operators and by Clark, Latushkin, Montgomery-Smith and Randolph for strongly continuous evolution families.

\section{Preliminaries}

Let $X$ be a Banach space, let $(\Theta, d)$ be a locally compact metric space and let $\mathcal{E}=X \times \Theta$. We denote by $\mathcal{B}(X)$ the Banach algebra of all bounded linear operators from $X$ into itself.

Definition 2.1. A continuous mapping $\sigma: \Theta \times \mathbf{R}_{+} \rightarrow \Theta$ is called a semiflow on $\Theta$, if $\sigma(\theta, 0)=\theta$, for all $\theta \in \Theta$ and

$$
\sigma(\theta, s+t)=\sigma(\sigma(\theta, s), t), \quad \forall(\theta, s, t) \in \Theta \times \mathbf{R}_{+}^{2} .
$$

Definition 2.2. A pair $\pi=(\Phi, \sigma)$ is called a linear skew-product semiflow on $\mathcal{E}=X \times \Theta$ if $\sigma$ is a semiflow on $\Theta$ and $\Phi: \Theta \times \mathbb{R}_{+} \rightarrow \mathcal{B}(X)$ satisfies the following conditions:

(i) $\Phi(\theta, 0)=I$, the identity operator on $X$, for all $\theta \in \Theta$; 
(ii) $\Phi(\theta, t+s)=\Phi(\sigma(\theta, t), s) \Phi(\theta, t)$, for all $(\theta, t, s) \in \Theta \times \mathbf{R}_{+}^{2}$ (the cocycle identity);

(iii) $(\theta, t) \mapsto \Phi(\theta, t) x$ is continuous, for every $x \in X$;

(iv) there are $M \geq 1$ and $\omega>0$ such that

$$
\|\Phi(\theta, t)\| \leq M e^{\omega t}, \quad \forall(\theta, t) \in \Theta \times \mathbf{R}_{+} .
$$

The mapping $\Phi$ given by Definition 2.2 is called the cocycle associated to the linear skew-product semiflow $\pi=(\Phi, \sigma)$. Examples of linear skew-product semiflows can be found in [4]-[6], [10], [11, [14]-20].

We denote by $\mathcal{C}_{s}(\Theta, \mathcal{B}(X))$ the space of all strongly continuous bounded mappings $H: \Theta \rightarrow \mathcal{B}(X)$.

Theorem 2.3. Let $\pi=(\Phi, \sigma)$ be a linear skew-product semiflow on $\mathcal{E}=X \times \Theta$. If $P \in \mathcal{C}_{s}(\Theta, \mathcal{B}(X))$ there is a unique linear skew product semiflow $\pi_{P}=\left(\Phi_{P}, \sigma\right)$ on $X \times \Theta$ such that

$$
\Phi_{P}(\theta, t) x=\Phi(\theta, t) x+\int_{0}^{t} \Phi(\sigma(\theta, s), t-s) P(\sigma(\theta, s)) \Phi_{P}(\theta, s) x d s
$$

for all $(x, \theta, t) \in X \times \Theta \times \mathbf{R}_{+}$.

Proof. See 14, Theorem 2.1.

Let $C\left(\mathbf{R}_{+}, X\right)$ be the linear space of all continuous functions $u: \mathbf{R}_{+} \rightarrow X$ and $C_{b}\left(\mathbf{R}_{+}, X\right)=\left\{u \in C\left(\mathbf{R}_{+}, X\right): \sup _{t \geq 0}\|u(t)\|<\infty\right\} . C_{b}\left(\mathbf{R}_{+}, X\right)$ is a Banach space with respect to the norm \|\|$u\|\|:=\sup _{t \geq 0}\|u(t)\|$.

Let $\pi=(\Phi, \sigma)$ be a linear skew-product semiflow on $\mathcal{E}=X \times \Theta$. For every $\theta \in \Theta$ we define the Perron operator

$$
P_{\theta}: C\left(\mathbf{R}_{+}, X\right) \rightarrow C\left(\mathbf{R}_{+}, X\right), \quad\left(P_{\theta} u\right)(t)=\int_{0}^{t} \Phi(\sigma(\theta, s), t-s) u(s) d s .
$$

Definition 2.4. A linear skew-product semiflow $\pi=(\Phi, \sigma)$ on $\mathcal{E}=X \times \Theta$ is called uniformly exponentially stable if there are $N \geq 1$ and $\nu>0$ such that

$$
\|\Phi(\theta, t)\| \leq N e^{-\nu t}, \quad \forall(\theta, t) \in \Theta \times \mathbf{R}_{+} .
$$

Definition 2.5. A linear skew-product semiflow $\pi=(\Phi, \sigma)$ on $\mathcal{E}=X \times \Theta$ is said to be uniformly $\left(C_{b}\left(\mathbf{R}_{+}, X\right), C_{b}\left(\mathbf{R}_{+}, X\right)\right)$-stable if

(i) $P_{\theta} u \in C_{b}\left(\mathbf{R}_{+}, X\right)$, for all $(u, \theta) \in C_{b}\left(\mathbf{R}_{+}, X\right) \times \Theta$;

(ii) there is $K>0$ such that $\left\|\mid P_{\theta} u\right\|\|\leq K\| u \|$, for all $(u, \theta) \in C_{b}\left(\mathbf{R}_{+}, X\right) \times \Theta$.

Theorem 2.6. Let $\pi=(\Phi, \sigma)$ be a linear skew-product semiflow on $\mathcal{E}=X \times \Theta$. Then $\pi$ is uniformly exponentially stable if and only if it is uniformly $\left(C_{b}\left(\mathbf{R}_{+}, X\right)\right.$, $\left.C_{b}\left(\mathbf{R}_{+}, X\right)\right)$-stable.

Proof. See [19], Theorem 5.1.

\section{Stability Radius}

If $U, Y$ are Banach spaces we denote by $\mathcal{B}(U, Y)$ the space of all bounded linear operators from $U$ into $Y$. If $\Theta$ is a locally compact metric space, we denote by 
$\mathcal{C}_{s}(\Theta, \mathcal{B}(U, Y))$ the space of all strongly continuous bounded mappings $H: \Theta \rightarrow$ $\mathcal{B}(U, Y)$, which is a Banach space with respect to the norm

$$
\|H\|:=\sup _{\theta \in \Theta}\|H(\theta)\| .
$$

Let $X, Y, U$ be Banach spaces, and let $\Theta$ be a locally compact metric space. Let $B \in \mathcal{C}_{s}(\Theta, \mathcal{B}(U, X))$ and $C \in \mathcal{C}_{s}(\Theta, \mathcal{B}(X, Y))$. Let $\pi=(\Phi, \sigma)$ be a linear skewproduct semiflow on $\mathcal{E}=X \times \Theta$.

Consider the system $S=(\pi, B, C)$ described by the following integral model:

$$
\left\{\begin{array}{l}
x\left(\theta, t, x_{0}, u\right)=\Phi(\theta, t) x_{0}+\int_{0}^{t} \Phi(\sigma(\theta, s), t-s) B(\sigma(\theta, s)) u(s) d s \\
y\left(\theta, t, x_{0}, u\right)=C(\sigma(\theta, t)) x\left(\theta, t, x_{0}, u\right)
\end{array}\right.
$$

where $t \geq 0,\left(x_{0}, \theta\right) \in \mathcal{E}$ and $u \in L_{l o c}^{1}\left(\mathbf{R}_{+}, U\right)$.

Definition 3.1. Suppose that $\pi=(\Phi, \sigma)$ is uniformly exponentially stable. The stability radius of $\pi$ with respect to the perturbation structure $(B, C)$ is defined by

$$
\begin{aligned}
r_{\text {stab }}(\pi, B, C) & =\sup \left\{r \geq 0: \forall \Delta \in \mathcal{C}_{s}(\Theta, \mathcal{B}(Y, U))\right. \text { with } \\
\|\Delta\| \leq r \Longrightarrow \pi_{B \Delta C} & \left.=\left(\Phi_{B \Delta C}, \sigma\right) \text { is uniformly exponentially stable }\right\} .
\end{aligned}
$$

In what follows we shall obtain a lower bound for the stability radius of $\pi$.

For every $\theta \in \Theta$ we define the bounded linear operators

$$
\begin{array}{ll}
B_{\theta}: C_{b}\left(\mathbf{R}_{+}, U\right) \rightarrow C_{b}\left(\mathbf{R}_{+}, X\right), & \left(B_{\theta} u\right)(t)=B(\sigma(\theta, t)) u(t), \\
C_{\theta}: C_{b}\left(\mathbf{R}_{+}, X\right) \rightarrow C_{b}\left(\mathbf{R}_{+}, Y\right), & \left(C_{\theta} u\right)(t)=C(\sigma(\theta, t)) u(t) .
\end{array}
$$

Let $\left\{P_{\theta}\right\}_{\theta \in \Theta}$ be the family of operators associated to $\pi$ according to relation (2.2). Since $\pi$ is uniformly exponentially stable, using Theorem 2.6, it follows that for every $\theta \in \Theta$ and every $u \in C_{b}\left(\mathbf{R}_{+}, U\right), C_{\theta} P_{\theta} B_{\theta} u \in C_{b}\left(\mathbf{R}_{+}, Y\right)$. Then it makes sense to consider the family of input-output operators $\left\{L_{\theta}\right\}_{\theta \in \Theta}$, where

$$
L_{\theta}: C_{b}\left(\mathbf{R}_{+}, U\right) \rightarrow C_{b}\left(\mathbf{R}_{+}, Y\right), \quad L_{\theta}=C_{\theta} P_{\theta} B_{\theta} .
$$

Using Theorem 2.6, we obtain that

$$
\alpha(\pi):=\sup _{\theta \in \Theta}\left\|L_{\theta}\right\|<\infty .
$$

In what follows we suppose that the mapping $C$ is uniformly positive, which means that there is $m>0$ such that

$$
\|C(\theta) x\|_{Y} \geq m\|x\|_{X}, \quad \forall(\theta, x) \in \Theta \times X .
$$

Theorem 3.2. Let $\Delta \in \mathcal{C}_{s}(\Theta, \mathcal{B}(Y, U))$ with

$$
\|\Delta\|<\frac{1}{\alpha(\pi)}
$$

Then there is $M \geq 1$ such that

$$
\left\|\Phi_{B \Delta C}(\theta, t)\right\| \leq M, \quad \forall(\theta, t) \in \Theta \times \mathbf{R}_{+} .
$$

Proof. For every $(x, \theta, t) \in \mathcal{E} \times \mathbf{R}_{+}$, let $M(x, \theta, t)=\sup _{s \in[0, t]}\left\|C(\sigma(\theta, s)) \Phi_{B \Delta C}(\theta, s) x\right\|$.

Let $\left(x, \theta, t_{0}\right) \in \mathcal{E} \times \mathbf{R}_{+}^{*}$. Let $\varepsilon>0$. We consider a continuous function $\alpha_{\varepsilon}: \mathbf{R}_{+} \rightarrow$ $[0,1]$ such that

$$
\alpha_{\varepsilon}(t)=1, \quad \forall t \in\left[0, t_{0}\right] \quad \text { and } \quad \alpha_{\varepsilon}(t)=0, \quad \forall t \geq t_{0}+\varepsilon .
$$


We define the function

$$
u_{\varepsilon}: \mathbf{R}_{+} \rightarrow X, \quad u_{\varepsilon}(t)=\alpha_{\varepsilon}(t) \Phi_{B \Delta C}(\theta, t) x .
$$

Then $u_{\varepsilon} \in C_{b}\left(\mathbf{R}_{+}, X\right)$. Using (2.1) for every $t \in\left[0, t_{0}\right]$, we have

$$
C(\sigma(\theta, t)) \Phi_{B \Delta C}(\theta, t) x=C(\sigma(\theta, t)) \Phi(\theta, t) x+\left(L_{\theta} \Delta_{\theta} C_{\theta} u_{\varepsilon}\right)(t) .
$$

If $N \geq 1$ is given by Definition 2.4, from the above relation we obtain

$$
\left\|C(\sigma(\theta, t)) \Phi_{B \Delta C}(\theta, t) x\right\| \leq\|C\| N\|x\|+\left\|L_{\theta}\right\|\|\Delta\|\left\|C_{\theta} u_{\varepsilon}\right\| \|, \quad \forall t \in\left[0, t_{0}\right] .
$$

We set $\delta=\alpha(\pi)\|\Delta\|$, and we obtain

$$
M\left(x, \theta, t_{0}\right) \leq\|C\| N\|x\|+\delta M\left(x, \theta, t_{0}+\varepsilon\right), \quad \forall \varepsilon>0 .
$$

Using the continuity of $\pi_{B \Delta C}$ we obtain that $M\left(x, \theta, t_{0}+\varepsilon\right) \longrightarrow M\left(x, \theta, t_{0}\right)$, as $\varepsilon \rightarrow 0$. Then, for $\varepsilon \rightarrow 0$ in (3.2) we deduce that

$$
M\left(x, \theta, t_{0}\right) \leq \frac{N\|C\|}{1-\delta}\|x\| .
$$

Let $m>0$ be given by (3.1). Then, from (3.3) we have that

$$
m\left\|\Phi_{B \Delta C}(\theta, t) x\right\| \leq \frac{N\|C\|}{1-\delta}\|x\|, \quad \forall t \in\left[0, t_{0}\right] .
$$

Since $x, \theta, t_{0}$ were arbitrary, we obtain

$$
\left\|\Phi_{B \Delta C}(\theta, t)\right\| \leq \frac{N\|C\|}{m(1-\delta)}, \quad \forall(\theta, t) \in \Theta \times \mathbf{R}_{+} .
$$

Theorem 3.3. For the system $S=(\pi, B, C)$ we have

$$
r_{\text {stab }}(\pi, B, C) \geq \frac{1}{\alpha(\pi)} .
$$

Proof. Let $\Delta \in \mathcal{C}_{s}(\Theta, \mathcal{B}(Y, U))$ with $\|\Delta\|<1 / \alpha(\pi)$. We set $\delta=\alpha(\pi)\|\Delta\|$. By Theorem 3.2, there is $M \geq 1$ such that $\left\|\Phi_{B \Delta C}(\theta, t)\right\| \leq M$, for all $(\theta, t) \in \Theta \times \mathbf{R}_{+}$. Let $(u, \theta) \in C_{b}\left(\mathbf{R}_{+}, X\right) \times \Theta$ and

$$
Q_{u, \theta}: \mathbf{R}_{+} \rightarrow X, \quad Q_{u, \theta}(t)=\int_{0}^{t} \Phi_{B \Delta C}(\sigma(\theta, s), t-s) u(s) d s .
$$

For every $n \in \mathbb{N}^{*}$ we consider a continuous function $\alpha_{n}: \mathbf{R}_{+} \rightarrow[0,1]$ with $\alpha_{n}(t)=1$, for all $t \in[0, n]$ and $\alpha_{n}(t)=0$, for all $t \geq n+1$. For every $n \in \mathbb{N}^{*}$ we define $u_{n}:=\alpha_{n} u$ and we consider

$$
Q_{u_{n}, \theta}: \mathbf{R}_{+} \rightarrow X, \quad Q_{u_{n}, \theta}(t)=\int_{0}^{t} \Phi_{B \Delta C}(\sigma(\theta, s), t-s) u_{n}(s) d s .
$$

For every $t \geq n+1$, using the cocycle identity, we obtain

$$
Q_{u_{n}, \theta}(t)=\Phi_{B \Delta C}(\sigma(\theta, n+1), t-n-1) \int_{0}^{n+1} \Phi_{B \Delta C}(\sigma(\theta, s), n+1-s) u_{n}(s) d s .
$$

It follows that

$$
\left\|Q_{u_{n}, \theta}(t)\right\| \leq M\left\|Q_{u_{n}, \theta}(n+1)\right\|, \quad \forall t \geq n+1
$$


so $Q_{u_{n}, \theta} \in C_{b}\left(\mathbf{R}_{+}, X\right)$. Using the relation (2.1), we obtain that

$$
\begin{gathered}
\left(C_{\theta} Q_{u_{n}, \theta}\right)(t)=\left(C_{\theta} P_{\theta} u_{n}\right)(t)+C(\sigma(\theta, t)) \int_{0}^{t} \int_{0}^{t-\tau} \Phi(\sigma(\theta, \tau+s), t-\tau-s) \\
B(\sigma(\theta, \tau+s)) \Delta(\sigma(\theta, \tau+s)) C(\sigma(\theta, \tau+s)) \Phi_{B \Delta C}(\sigma(\theta, \tau), s) u_{n}(\tau) d s d \tau \\
=\left(C_{\theta} P_{\theta} u_{n}\right)(t)+C(\sigma(\theta, t)) \int_{0}^{t} \int_{\tau}^{t} \Phi(\sigma(\theta, \xi), t-\xi) \\
B(\sigma(\theta, \xi)) \Delta(\sigma(\theta, \xi)) C(\sigma(\theta, \xi)) \Phi_{B \Delta C}(\sigma(\theta, \tau), \xi-\tau) u_{n}(\tau) d \xi d \tau .
\end{gathered}
$$

Using Fubini's theorem it follows that

$$
\begin{gathered}
\left(C_{\theta} Q_{u_{n}, \theta}\right)(t)=\left(C_{\theta} P_{\theta} u_{n}\right)(t)+C(\sigma(\theta, t)) \int_{0}^{t} \int_{0}^{\xi} \Phi(\sigma(\theta, \xi), t-\xi) \\
B(\sigma(\theta, \xi)) \Delta(\sigma(\theta, \xi)) C(\sigma(\theta, \xi)) \Phi_{B \Delta C}(\sigma(\theta, \tau), \xi-\tau) u_{n}(\tau) d \tau d \xi \\
=\left(C_{\theta} P_{\theta} u_{n}\right)(t)+\left(C_{\theta} P_{\theta} B_{\theta} \Delta_{\theta} C_{\theta} Q_{u_{n}, \theta}\right)(t), \quad \forall t \geq 0 .
\end{gathered}
$$

It follows that

$$
\left\|C_{\theta} Q_{u_{n}, \theta}(t)\right\| \leq\|C\| K\left\|u_{n}\right\|\left|+\delta\left\|C_{\theta} Q_{u_{n}, \theta}\right\|\right|, \quad \forall t \geq 0
$$

where $K=\sup _{\theta \in \Theta}\left\|P_{\theta}\right\|$. Thus, we deduce that

$$
\left\|C_{\theta} Q_{u_{n}, \theta}\right\|\left|\leq \frac{K\|C\|}{1-\delta}\|\| u \|\right|, \quad \forall n \in \mathbb{N}^{*} .
$$

If $m>0$ is given by (3.1), then we obtain that

$$
m\left\|Q_{u_{n}, \theta} \mid\right\| \leq \frac{K\|C\|}{1-\delta}\|\| u\|\|, \quad \forall n \in \mathbb{N}^{*} .
$$

Since for $t>0$ and $n=[t]+1$ we have that $Q_{u_{n}, \theta}(t)=Q_{u, \theta}(t)$, using the above inequality, we obtain that

$$
\left\|\left|Q_{u, \theta}\right|\right\| \leq \frac{K\|C\|}{m(1-\delta)} \mid\|u\| \|, \quad \forall(u, \theta) \in C_{b}\left(\mathbf{R}_{+}, X\right) \times \Theta .
$$

Then by Theorem 2.6, we conclude that $\pi_{B \Delta C}$ is uniformly exponentially stable, which ends the proof.

\section{REFERENCES}

1. J. A. Ball, I. Gohberg, and M. A. Kaashoek, Two-sided Nudelman interpolation for inputoutput operators of discrete time-varying systems, Integral Equations Operator Theory 21 (1995), 174-211. MR 95m:47018

2. J. A. Ball, I. Gohberg, and M. A. Kaashoek, Input-output operators of J-unitary time-varying continuous time systems, Oper. Theory Adv. Appl. 75 (1995), 57-94. MR 96c:47109

3. J. A. Ball, I. Gohberg, and M. A. Kaashoek, A frequency response function for linear, timevarying systems, Math. Control Signals Systems 8 (1995), 334-351. MR 97h:93014

4. C. Chicone and Y. Latushkin, Evolution Semigroups in Dynamical Systems and Differential Equations, Mathematical Surveys and Monographs, vol. 70, Amer. Math. Soc., Providence, RI, 1999. MR 2001e:47068

5. S.-N. Chow and H. Leiva, Existence and roughness of the exponential dichotomy for linear skew-product semiflows in Banach space, J. Differential Equations 120 (1995), 429-477. MR 97a:34121

6. S.-N. Chow and H. Leiva, Unbounded perturbation of the exponential dichotomy for evolution equations, J. Differential Equations 129 (1996), 509-531. MR 98d:34090 
7. S. Clark, Y. Latushkin, S. Montgomery-Smith, and T. Randolph, Stability radius and internal versus external stability in Banach spaces: an evolution semigroup approach, SIAM J. Control Optimization 38 (2000), 1757-1793. MR 2001k:93085

8. D. Hinrichsen, A. Ilchmann, and A. J. Pritchard, Robustness of stability of time-varying linear systems, J. Differential Equations 82 (1989), 219-250. MR 91b:93122

9. D. Hinrichsen and A. J. Pritchard, Robust stability of linear operators on Banach spaces, SIAM J. Control Optim. 32 (1994), 1503-1541. MR 95i:93109

10. Y. Latushkin, S. Montgomery-Smith, and T. Randolph, Evolutionary semigroups and dichotomy of linear skew-product flows on spaces with Banach fibers, J. Differential Equations 125 (1996), 73-116. MR 97a:47056

11. Y. Latushkin and R. Schnaubelt, Evolution semigroups, translation algebras and exponential dichotomy of cocycles, J. Differential Equations 159 (1999), 321-369. MR 2000k:47054

12. M. Megan, B. Sasu, and A. L. Sasu, On nonuniform exponential dichotomy of evolution operators in Banach spaces, Integral Equations Operator Theory 44 (2002), 71-78. MR 2003d:47058

13. M. Megan, A. L. Sasu, and B. Sasu, Discrete admissibility and exponential dichotomy for evolution families, Discrete Contin. Dynam. Systems 9 (2003), 383-397. MR 2003k:47054

14. M. Megan, A. L. Sasu, and B. Sasu, Stabilizability and controllability of systems associated to linear skew-product semiflows, Rev. Mat. Complutense 15 (2002), 599-618. MR 2004b:93114

15. M. Megan, A. L. Sasu, and B. Sasu, Theorems of Perron type for uniform exponential dichotomy of linear skew-product semiflows, Bull. Belg. Math. Soc. Simon Stevin 10 (2003), $1-21$.

16. M. Megan, A. L. Sasu, and B. Sasu, Perron conditions for uniform exponential expansiveness of linear skew-product flows, Monatsh. Math. 138 (2003), 145-157. MR 2003k:47055

17. M. Megan, A. L. Sasu, and B. Sasu, Perron conditions for pointwise and global exponential dichotomy of linear skew-product flows, accepted for publication in Integral Equations Operator Theory.

18. M. Megan, A. L. Sasu, and B. Sasu, Uniform exponential dichotomy and admissibility for linear skew-product semiflows, Oper. Theory Adv. Appl. (2003).

19. M. Megan, A. L. Sasu, and B. Sasu, Theorems of Perron type for uniform exponential stability of linear skew-product semiflows, accepted for publication in Dynam. Contin. Discrete Impuls. Systems.

20. R. J. Sacker and G. R. Sell, Dichotomies for linear evolutionary equations in Banach spaces, J. Differential Equations 113 (1994), 17-67. MR 96k:34136

Department of Mathematics, Faculty of Mathematics and Computer Science, West University of Timişoara, Romania

E-mail address: sasu@math.uvt.ro

Department of Mathematics, Faculty of Mathematics and Computer Science, West University of TimişoAra, Romania

E-mail address: 1bsasu@yahoo.com 